\title{
El lugar de la filosofía de la agronomía dentro del campo de la filosofía de las ciencias de la vida*
}

The Place of Philosophy of Agronomy in the Field of Philosophy of Life Sciences

Francisco Javier Serrano-Bosquet

Eva Luisa Rivas Sada ${ }^{\dagger \ddagger}$

\begin{abstract}
Resumen
El objetivo principal de este trabajo es llamar la atención sobre la necesidad de establecer colegiada y formalmente las bases constitutivas de una nueva rama de la filosofía de las ciencias de la vida, una filosofía de la agronomía, que dé cuenta de los fenómenos específicos de esta disciplina, a la vez que proponer algunos principios que, como ideas detonadoras, funjan como bases iniciales sobre las que pueda levantarse esta filosofía centrada. Comenzamos viendo por qué, a pesar de haber ya algunos trabajos sobre agronomía en los que se abordan cuestiones epistemológicas, ontológicas y éticas, aún no ponemos hablar de una filosofía de la agronomía formalmente constituida, por qué los estudios sociales de la ciencia y la tecnología no son suficientes para alcanzar una profunda comprensión del fenómeno agronómico y cuáles son algunos elementos básicos que se deberían contemplar en este nuevo campo filosófico. A continuación mostramos por qué estimamos que se trataría de una filosofía genitiva que debería desarrollarse en torno a dos tipos de modulaciones, una objetiva y otra subjetiva, y en qué consistiría cada una de ellas. Finalizamos apuntando algunos de los primeros pasos que estimamos deberían darse y las metas que a largo plazo nos gustaría que, como comunidad, se pudieran alcanzar.
\end{abstract}

Palabras clave: filosofía de la agronomía - producción agraria - filosofía de la ciencia

\begin{abstract}
The main goal of this paper is to call attention on the need to establish, in a formal and collegiate manner, the foundations of a Philosophy of Agronomy as a new branch stemming from the Philosophy of Life Sciences. This paper proposes a set of principles that could function as common ground onto which this centered philosophy could develop. We begin by analyzing why, despite the presence of several works on agronomy that approach to epistemological, ontological, and ethical matters, we cannot talk about a formally constituted Philosophy of Agronomy. We also address the issue of why social studies on science and technology are insufficient in order to achieve a deep understanding of the agronomic phenomenon, and inquire about the basic elements to consider this new philosophical field. Additionally, we show why we consider it a sort of genitive philosophy which should develop around two types of modulations, an objective and a subjective one, and we explain both of them. Lastly, we propose some of the first steps to be taken and some of the long-term goals that we wish our academic community to achieve.
\end{abstract}

Keywords: philosophy of agronomy - agricultural production - philosophy of science

\footnotetext{
* Recibido: 30 de Junio de 2014. Aceptado en versión revisada: 13 de Agosto de 2014.

† Tecnológico de Monterrey, México. Para contactar a los autores, por favor, escribir a: fjavierserrano@itesm.mx o evarivassada@gmail.com.

* Este estudio forma parte del proyecto de investigación titulado "La influencia de la concepción de ciencia de la Fundación Rockefeller en el desarrollo de la investigación biológica y agrícola mexicana" [número 168062] financiado por CONACYT en su convocatoria de 2011 de Investigación Básica SEP-CONACYT, Modalidad Joven Investigador (J2). Queremos dar las gracias a los directores y personal de la Biblioteca Cervantina del Tecnológico Monterrey, la Biblioteca de Ciencias de la UNAM y la Biblioteca de Ciencias de la Tierra de la UNAM por las facilidades que nos dispensaron durante las estancias alli llevadas a cabo y, sobre todo, por su amabilidad y excelente disposición en todo momento.

Metatheoria 5(1)(2014): 175-186. ISSN 1853-2322.

(c) Editorial de la Universidad Nacional de Tres de Febrero. Publicado en la República Argentina.
} 


\section{Introducción}

El objetivo principal de este trabajo es llamar la atención sobre la necesidad de articular colegiada y formalmente las bases constitutivas de una nueva rama de la filosofía de las ciencias de vida, la filosofía de la agronomía, y proponer algunos principios que, como ideas detonadoras, funjan como bases iniciales sobre las que pueda levantarse esta filosofía genitiva. Es decir, no se trata de dar una respuesta completa, totalmente articulada de la misma, sino de empezar a crear las condiciones para el diálogo compartido. De ahí, que no señalemos un único marco teórico y metodológico concreto desde el que poner en marcha este proyecto, entendemos que no es momento de restar, sino de sumar iniciativas, propuestas y perspectivas a un nuevo campo de estudio. Comenzamos, para ello, constatando la inexistencia de una filosofía de la agronomía formalmente constituida y señalando tanto la pertinencia de su existencia como algunas de sus principales características distintivas frente a aquellas otras disciplinas que, como los estudios sociales y culturales de la ciencia, parecen estar haciéndose cargo ya de las mismas cuestiones. Posteriormente describimos los dos caminos que, en principio y a nuestro juicio, entendemos como principales y sobre los cuales podría vertebrarse este nuevo campo. Concluimos con una primera propuesta formal de trabajo y reflexión -que es lo que en esta convocatoria entendemos se pide- colegiada.

\section{La inexistencia de la filosofía de la agronomía y una primera caracterización posible}

Cualquier persona que haya intentado acercarse al estudio de la agricultura habrá podido comprobar la vasta bibliografía existente en torno a la misma y cómo desde la economía, la antropología y geografía social, el derecho, la política e incluso la polemología se ha estado y se está preguntando por cuestiones relacionadas con la producción de alimentos y materias primas. Lo mismo ocurre en torno a los retos ecológicos y sociales actuales que se nos presentan ante el profundo desequilibrio gestado a partir de una nueva relación entre el ser humano y naturaleza en el seno de la sociedad contemporánea. Ahora bien, ¿qué ha dicho la filosofía en general y la filosofía de la ciencia en particular al respecto? Encontramos, desde luego, algunos trabajos importantes llevados a cabo desde nuestra disciplina, pero se han tratado principalmente de esfuerzos individuales que han llevado casi circunstancialmente a diversos autores a tratar cuestiones epistemológicas, metodológicas y éticas de manera aislada y autónoma alrededor de proyectos o programas concretos de agricultura, ciertas ciencias o determinadas prácticas agronómicas. Es el caso, por ejemplo, de los trabajos de Dan Binkley y Richard F. Fisher (2013), Ecology and Management of Forest Soils; R. Paul Thompson (2011), Agro-Technology: A Philosophical Introduction; Billie R. DeWalt (1978), Appropriate Technology in Rural Mexico: Antecedents and Consequences of an Indigenous Peasant Innovation, o nuestro mismo trabajo "Warren Weaver y el Programa de Biología Experimental de la Fundación Rockefeller" (Serrano-Bosquet \& Caponi 2014). Si bien se trata de interesantes trabajos donde se abordan cuestiones filosóficas relacionadas con o inscritas en el contexto de la producción agraria, se trata de trabajos aislados, fuera de los ámbitos y circuitos más comunes de la filosofía de la ciencia. Tanto éstos como la gran mayoría de trabajos de este tipo localizados han aparecido publicados en revistas o colecciones y series de historia agraria, de ética ambiental, de sociología rural, de políticas públicas para el campo, de biotecnología o de economía agrícola. Ello nos da una primera idea de la posible dispersión de los trabajos filosóficos en el área e invita a hacer revisiones y análisis de contenido en publicaciones no estricta u originalmente filosóficas. En ese sentido una primera aproximación no excesivamente formal arroja ya las primeras evidencias de ello. Es el caso, por ejemplo, de los artículos de Thelma Lazo-Flores (2012), "Grading green: Strands of Sustainability Theory and Practice in Interior Design Courses"; Nancy E. Shurtz (2012), "Eco-Friendly Building from the Ground up: Environmental Initiatives and the Case of Portland, Oregon"; Dane Scott (2011), "The Technological Fix Criticisms and the Agricultural 
Biotechnology Debate"; Anthony Alexander (2008), "Different Paths, same Mountain: Daoism, Ecology and the New Paradigm of Science"; Andrew Light y Aurora Wallace (2005), "Not out of the Woods: Preserving the Human in Environmental Architecture"; M. Trias (1987), "Population and Development"; Lucy Carter (2007), "A Case for a Duty to Feed the Hungry: GM Plants and the third World"; Johann A. Klaassen (2007), "Contemporary Biotechnology and the new "Green Revolution": Feeding the World with "Frankenfoods"?"; Kristin Shrader-Frechette (2005), "Property Rights and Genetic Engineering: Developing Nations at Risk"; Robert W. Loftin (1993), "The Violence of the Green-Revolution - Third World Agriculture, Ecology, and Politics - Shiva,V”; Amelie Berger (1995), "Is Agricultural Research in Africa Worthwhile today?"; Lester R. Brown (1981), "World Food Resources and Population: The Narrowing Margin”. Trabajos todos ellos que, si bien tocan aspectos que podríamos considerar propios (no necesariamente exclusivos) de la filosofía de la ciencia y la agronomía, ni se identifican como trabajos de filosofía de la agronomía ni aparecen en publicaciones filosóficas.

Si llevamos a cabo una búsqueda en las bases de datos Scopus e Isiweb a partir del término Filosofía de la Agronomía (y su correspondiente en inglés Philosophy of Agronomy) tan sólo aparecen cerca de una treintena de trabajos, de los cuales, tan sólo un tercio pertenecen al área de humanidades y ninguno puede ser considerado como un verdadero trabajo de filosofía de la agronomía, ni siquiera de la agricultura. Si bien a aparecen como tal una serie de artículos dedicados al estudio y análisis (varias veces comparativo) de historiadores naturales como Erasmus y Charles Darwin, Linneo y Francis Galton, el análisis de contenido los descarta inmediatamente. Otros trabajos, como el texto de Frank N. Egerton (2012), Roots of Ecology. Antiquity to Haeckel, que también aparece en el listado tampoco puede considerarse un trabajo de filosofía de la agricultura. Se trata de una muy interesante obra sobre la extraordinaria importancia y el papel que ha jugado la ecología en muchas de las decisiones que ha tenido que tomar la humanidad, al presentar ante el lector un recorrido histórico sobre los conceptos e ideas ecológicas; empero, no hay un abordaje de cuestiones agrícolas o agrarias desde un punto de vista filosófico. Por su parte, Samantha Noll (2013) lleva a cabo en su texto "Broiler Chickens and a Critique of the Epistemic Foundations of Animal Modification" un trabajo muy interesante desde el punto de vista filosófico, pero que aborda la cuestión avícola, no la agronómica. Ahora bien, este trabajo nos da una pista muy interesante: el artículo está publicado en la revista Journal of Agricultural and Environmental Ethics, una publicación en la que ya empezamos a ver trabajos en la línea que aquí planteamos. Lo mismo podríamos decir del libro de J. Clarke (2010), The Ordinary People of Essex: Environment, Culture, and Economy on the Frontier of Upper Canada. En este trabajo Clarke hace una excelente descripción y análisis histórico del uso de las tierras en el Alto Canadá y la influencia de la actividad agrícola en la región: el seguimiento de los éxitos y fracasos de las prácticas comunes de la tierra llevados a cabo por los colonos del condado de Essex, se realiza a partir de un notable número de temas y factores, desde geográficos, energéticos o biológicos, hasta económicos y culturales. El trabajo de B.R. Cohen (2009), Notes from the Ground: Science, Soil, and Society in the American Countryside, examina en una línea muy parecida a la de Clarke las condiciones culturales bajo las cuales llegaron la agricultura y la ciencia a la América del siglo XIX. A través de la integración de la historia de la ciencia, la historia ambiental y los estudios sociales de la ciencia, el libro muestra cómo y por qué los agricultores, terratenientes y responsables políticos estadounidenses se resistieron, inicialmente, a implementar conocimientos y métodos científicos a la hora de trabajar la tierra. Asimismo, Cohen muestra cómo la credibilidad en las nuevas prácticas sobre el uso del suelo no vino de la mano de los químicos, sino gracias a la ideología existente sobre el trabajo, el conocimiento y la ciudadanía.

Queremos finalizar este pequeño análisis bibliográfico con el texto de P.B. Thompson (2008), The Agricultural Ethics of Biofuels: A First Look. Un documento que es, en sí mismo, una reflexión preliminar para el establecimiento de un nuevo tipo de investigación filosófica, una filosofía de la agronomía. Para tal fin pone sobre la mesa dos tipos de enfoques filosóficos que deben estar presentes dentro de esta filosofía. En primer lugar, un análisis crítico de los compromisos epistemológicos subyacentes en los modelos científicos sobre los que se sustenta un proyecto o programa; y un segundo enfoque sobre el 
sentido y posicionamiento que dicho proyecto tiene dentro de la misma, desde una perspectiva fundamentalmente ética.

¿Podemos a partir de estas y otras referencias hablar ya de una filosofía de la agronomía formalmente constituida? Si bien el número de trabajos existentes así como su dispersión parecen desde un inicio decir que no, el término "filosofía de la agronomía" ha sido utilizado, hasta la fecha, de manera poco recurrente, articulada y mucho menos generalizada, uno de los principales criterios externos o indicadores sociológicos propuesto por Alejando Cassini (2013, pp. 73-74) para intentar establecer la fecha de inicio de una determinada disciplina científica. Tampoco encontramos sociedades o asociaciones de filosofía o de estudios sociales y culturales de la agronomía, ni congresos específicos sobre la temática o publicaciones especializadas, cátedras y cursos profesionales de filosofía de la agronomía ni, por supuesto, libros de texto ni apoyo para ellos; criterios todos ellos señalados por el filósofo argentino como fundamentales para comenzar a hablar de la constitución formal de una disciplina. De ahí que no podamos, por consiguiente, considerar la existencia de una filosofía formal de la agronomía.

A la luz de la inexistencia de esta filosofía formal y a raíz de las investigaciones llevadas a cabo en los últimos años, de la bibliografía consultada y, sobre todo, del actual proyecto de investigación que estamos llevando a cabo en torno al papel que la Fundación Rockefeller jugó en el desarrollo de la investigación agronómica en México, consideramos que ha llegado el momento de dar un salto importante, de ir más allá del marco general que nos ofrece la filosofía de las ciencias de la vida o de la biología y constituir, dentro de o junto a estas, una filosofía de la agronomía. Una filosofía que debería ser considerada -al menos en principio- como una parte de la filosofía de las ciencias de la vida y cuya metodología y forma de análisis estaría estrechamente relacionadas con la filosofía de la biología. Ahora bien, pese a seguir habiendo cierto grado de dependencia, debería ir tomando forma también fuera de las mismas, ya que el fenómeno agrario desborda el biológico y adquiere otra forma de complejidad. Baste para darse cuenta de ello la diversidad de ciencias de las que se alimenta la agronomía; una diversidad que apunta, en principio, a pensar en una filosofía genitiva objetiva, es decir, una disciplina de segundo orden que tendría por objeto material de estudio las ciencias e ingenierías agronómicas. En esta tesitura sería necesario, al menos, la integración de datos y conceptos de los diferentes niveles de complejidad y ámbitos como la biología vegetal, la fitopatología, la entomología, la edafología, la climatología, la geohidrología, entre otras ramas que estudian los diversos fenómenos naturales que inciden en el ciclo vital de los cultivos en explotación. La agricultura, su práctica concreta y significado, vendría a ser objeto de estudio en un segundo momento, precisamente a la luz de los conocimientos y datos aportados por esta primera aproximación. Empero, insistimos, eso sería insuficiente pues en este segundo análisis, ya sobre la agricultura, debieran además analizarse los datos y conceptos aportados, al menos, desde la economía agrícola, la sociología rural, la antropología social y la historia agraria que pretenden comprender a la sociedad rural en toda su complejidad cultural. ¿Por qué lo pensamos así? Porque la agronomía tiene por objeto de estudio a la agricultura y ésta no es una manifestación de la naturaleza, es un constructo cultural, el principio civilizatorio de la humanidad. Sólo a partir de estos dos momentos, entendemos, sería posible el análisis y la comprensión totalizadora -como cabría suponer que corresponde a la filosofía- de la cuestión agraria. En pocas palabras, comprendería parte de las distintas disciplinas de las ciencias (sobre el orden natural y cultural) que abordan el complejo fenómeno de los agroecosistemas desarrollados por el hombre. La identificación en cada caso de los principales campos y dominios desde los que nutrirse conceptualmente, de los agentes participantes, valores, bienes e intereses que están en juego en cada caso de estudio; los marcos ideológicos, axiológicos y por supuesto epistemológicos y metodológicos es y será -entendemos- fundamental para los propósitos de esta nueva vertiente filosófica. En cualquier caso, consideramos pertinente, en este primer esfuerzo, delinear con algo más de claridad los contornos del campo de estudio de la filosofía de la agronomía, es decir de la propia agronomía, de tal forma que nos permita abrir la discusión sobre su posible naturaleza. 
Como hemos mencionado líneas arriba, la filosofía de la agronomía se encontraría en parte ubicada al interior de la filosofía de las ciencias de la vida, como un subcampo que tendría como objeto de estudio las especies y variedades domesticadas y bajo explotación por el hombre; dicho de otro modo, un campo particular que intentaría comprender una de las rutas de interacción entre el hombre y la naturaleza. En ese sentido, su tradición sería tan remota como la propia agricultura. No obstante, creemos que esto está por debatirse, pues la agronomía que se nos presenta al día de hoy, tal cual como la conocemos, es una ciencia moderna, un campo científico autónomo y de gran complejidad por su naturaleza interdisciplinaria (tecnocientífica). Y estas dos características fundamentales se adquirieron en tiempos mucho más recientes. Consideramos que estos rasgos distintivos son el resultado de un proceso histórico particular: resulta ser que, en ciertos momentos y lugares determinados, este rico acervo de conocimientos científicos fue sustituyendo lenta y paulatinamente a los saberes empíricos tradicionales en el propio quehacer agrícola. Dicho desde una perspectiva más amplia, obedecen a la creciente articulación entre las esferas de la investigación científica, del desarrollo tecnológico y de las propias actividades agropecuarias; cuya interacción dinámica gestaron, al mismo tiempo, a la ingeniería agronómica, la nueva profesión científica al servicio del campo, y a una nueva modalidad de agricultura comercial reconocida como agricultura científica. En pocas palabras, la agronomía y la agricultura científica son un mismo fenómeno con dos caras. Consideramos que este proceso histórico tan representativo de las sociedades desarrolladas se dio a partir de la segunda mitad del siglo XIX y formó parte de la creciente especialización científica y, por ende, del desmembramiento de la Historia Natural. Por lo tanto, su corporeidad y autonomía en el ámbito de las ciencias de la vida, así como su confluencia con el desarrollo ingenieril se expresa ya con claridad al concluir el siglo XIX, particularmente con la eclosión de las nuevas prácticas culturales típicamente características del extraordinario desarrollo de la agricultura científica norteamericana. Pero no sólo a ello. La creciente complejidad de su campo de estudio se debe comprender también como parte esencial del fenómeno tecno-económico de la economía industrial (el capitalismo clásico), cuya mejor expresión se realiza desde el siglo pasado. En sus primeras décadas, la agronomía amplió sus alcances al integrar aquellas disciplinas provenientes del campo de la cultura, dedicadas a estudiar el fenómeno de la sociedad rural, en una época convulsa de reclamos y reformas sobre la "cuestión social”. Al cubrirse todas las aristas posibles de la relación hombre-naturaleza fue entonces cuando pudo abordarse en toda su complejidad a los agroecosistemas conformados por la acción humana. Éste resultado, desde su traducción ingenieril, proyectó a la agricultura comercial de base científica de tal forma y magnitud que ha desatado graves problemas ecológicos y sociales, pese a, o debido a que, se alcanzó un crecimiento sostenido de la productividad y una mayor disposición de alimentos a nivel mundial, como nunca antes. De ahí la abundancia actual de investigaciones de todo tipo y debates polémicos en torno a éste específico modelo científico de la ingeniería agronómica, y a la propia agricultura científica, tan productiva como destructora. En consecuencia, si atendemos su conformación histórica como campo interdisciplinario y autónomo, y al gran interés despertado en la esfera intelectual en las últimas décadas, debemos al menos ubicar a la agronomía como una de las manifestaciones más acabadas y contradictorias de la cultura contemporánea y, por lo tanto, la filosofía de la agronomía estaría delineada por estas características culturales espaciotemporales y a ella se debería. Pero eso está por debatirse. Ahora bien, ¿en qué consistiría su quehacer?

\section{Los dos caminos de la filosofía de la agronomía}

Nuestra propuesta parte de la convicción de que la filosofía de la agronomía, como toda filosofía de la ciencia, debe ser útil -como señalaría Mario Bunge- a la sociedad, a la ciencia y a la misma filosofía (Bunge 2004, p. 28), como veremos el líneas posteriores. Por lo pronto y como principal propósito, consideramos que debe facilitar una serie de conocimientos y herramientas conceptuales (y metodológicas) que permitan la comprensión del fenómeno agronómico y agrario en sí; pero, sobre todo, de los fenómenos y proyectos agrícolas concretos. ¿Cómo sería ello posible? 
$\mathrm{Al}$ investigador se le presentan dentro de la filosofía de la agronomía dos grandes caminos o áreas no excluyentes sino complementarias. El primero de ellos partiría -tal y como ya hemos adelantadode la identificación de las distintas ciencias e ingenierías que participan en el desarrollo de la agronomía y observaría la forma en la que lo hacen. De ese modo, los conceptos, teorías y datos presentados como propios o procedentes de estas disciplinas conformarían el primer conjunto de objetos (materiales) de estudio. Además de ello, y con el propósito de lograr una mejor comprensión en esta primera aproximación, debieran incluirse también como objetos de estudio las preguntas, conceptos, reflexiones e ideas de las filosofías centradas de cada una de estas disciplinas; es decir, también serían objeto de estudio y material de trabajo, las aportaciones de la filosofía de la biología, la física, la filosofía política, etc. A partir de estos materiales, pero no exclusivamente, podríamos empezar a poner en marcha proyectos concretos de investigación que, en un primer momento (no necesariamente habría que seguir este orden), siguieran líneas de investigación clásicas. Es decir, fungiría como una suerte de criteriología de segundo orden que, teniendo como objeto de estudio las ciencias e ingenierías agronómicas, tuviera como primer y principal objetivo poner al descubierto cuestiones epistemológicas, ontológicas y metodológicas. Vendría a ser, en términos de Gustavo Bueno (2004), una suerte de filosofía genitiva objetiva en cuanto a que el objeto de estudio serían los conceptos, los datos, teorías y conocimientos procedentes de las distintas ciencias, ingenierías y tecnologías agronómicas; en términos de Hugh Lacey sería un acercamiento a los valores cognitivos de esta práctica o de las disciplinas que participan en la misma (Lacey 1998); o utilizando la propuesta ecológica de Naess, una aproximación superficial (shallow) al fenómeno agrícola.

Es probable que algunos autores consideren este primer paso o proceso un tanto anticuado, superado en cuanto que les recuerde los programas positivistas de inicios del siglo XX; sin embargo, entendemos que es fundamental, si bien no suficiente, intentar identificar las principales disciplinas y áreas científicas que participan en el desarrollo agronómico. $\mathrm{O}$ al menos abordarlas desde finales del siglo XIX, cuando disciplinas como la física, la química, la biología y la meteorología se transformaron en elementos constitutivos de la investigación agronómica misma. Colocarlas como objeto principal de estudio, daría vida a esta filosofía genitiva objetiva. Este camino debiera ayudarnos, finalmente, a identificar las principales (aunque no exclusivas) aproximaciones filosóficas (epistemológicas, axiológicas, etc.) que deberían en principio considerarse como propias de la filosofía de la agronomía.

El segundo camino o tipo de aproximación nos permitiría llevar a cabo aproximaciones más profundas (deep) -en términos nuevamente de Naess-, e ir más allá de las revisiones conceptuales, teóricas, epistemológicas u ontológicas, poniendo al descubierto los valores no cognitivos (volviendo a Lacey), al identificar, por ejemplo, a los principales agentes que participan en los orígenes, la naturaleza, la identidad propia, la lógica, etc. de los proyectos agronómicos concretos a estudiar. Dando un paso más allá, este segundo camino parte de la convicción de que la actividad agronómica es una actividad sumamente compleja y que debe ser abordada como tal, desde la multiplicidad de perspectivas, áreas y metodologías. Trayendo a nuestro terreno la propuesta de Alberto Elena y Javier Ordoñez, entendemos que el desarrollo científico y tecnológico no se da al margen de la sociedad y cultura en la que se desarrolla. En palabras de estos autores:

Fue la misma sociedad de Europa occidental -apunta Peter Mathias- la que asistió a los grandes avances de la ciencia y la tecnología a lo largo del vasto lapso temporal que va del siglo XV al siglo XVIII. Considerar esto como un accidente sería erigir el nihilismo como dogma (Elena \& Ordóñez 1996, p. 543).

Del mismo modo, el nacimiento de la agronomía -y por extensión de la agricultura científica- no se puede entender al margen de la cultura y sociedad en la que se produce, de los valores e intereses, y de las perspectivas de los agentes que participan directa o indirectamente en su desarrollo.

Nada nuevo decimos con ello, pero entendemos que es oportuno recordar que este es un problema no exclusivo de la pretendida filosofía de la agronomía, sino de la filosofía general de la ciencia y de las distintas filosofías particulares o centradas: en ninguno de estos casos el análisis lógico y normativo de las teorías, así como su reconstrucción racional, es suficiente. Tomando la clásica distinción 
popularizada por Reichenbach en 1938 -si bien ya aceptada desde mucho tiempo antes y rehabilitada en el último cuarto del siglo XX (Cassini 2013, pp. 70-71)- entre contexto de justificación y contexto de descubrimiento, la filosofía de la agronomía debería hacerse cargo también de los aspectos sociales y culturales presentes y condicionantes de todo proyecto agronómico. Siguiendo a Cassini (2013), entendemos que a pesar de las enormes críticas recibidas por el enfoque historicista de la filosofía de la ciencia, la rehabilitación del contexto de descubrimiento como campo accesible al análisis filosófico, da entrada a los factores históricos y "externos", relegados por los empiristas lógicos, como elementos fundamentales para el estudio filosófico del o de los fenómenos agronómicos. Si es cierto que no todos los filósofos de la ciencia se volvieron en su momento ni son hoy propiamente historicistas, si bien no debemos ignorar las fuertes críticas al llamado "relativismo socio-epistémico", lo cierto es que casi todos los trabajos de filosofía de la ciencia actuales incluyen algún caso histórico y casi todos los filósofos reconocen su relevancia para la filosofía de la ciencia. La cuestión que está aún en discusión es hasta dónde deberían llegar las aportaciones históricas, así como del resto de factores o elementos del llamado contexto de descubrimiento para poder seguir hablando de filosofía de la ciencia (o en nuestro caso filosofía de la agronomía). Esta cuestión no es baladí, ya que es muy posible que haya quienes compartan la preocupación e interés por las problemáticas planteadas, pero que sostengan a la vez que, llegados a este punto, se abandona el terreno de la filosofía de la ciencia adentrándonos, por consiguiente, en el de los llamados estudios sociales o culturales de la ciencia. Ello, lejos de ser un problema, es en principio una buena señal, ya que constata la necesidad y pertinencia de este tipo de estudios más allá de las etiquetas disciplinarias que queramos poner. En cualquier caso, ¿cabe aún algún lugar a una filosofía de la agronomía?

Si bien no pretendemos dar una repuesta definitiva, ya que entendemos que esta es una cuestión a discutir gremialmente (junto a otras filosofías de las ciencias centradas), juzgamos -tal y como veremos de manera un tanto más extendida más adelante- que la existencia de los estudios sociales y culturales de la ciencia no agotan completamente la temática. Por el contrario, entendemos que muchos de ellos apuntan hacia la necesidad de una filosofía centrada, de una filosofía de la agronomía que junto a los factores contextuales, profundice en las cuestiones y fundamentos epistemológicos, cognoscitivos y metodológicos constitutivos de la misma agronomía, así como en los conflictos éticos que están de fondo. Es decir, apuntan hacia la posibilidad y necesidad de una filosofía de la agronomía que puede y debe, por ser genitiva, entendida, interpretada y desarrollada en sus dos formas. La primera de ellas, de la que ya hemos dado cuenta, sería una filosofía genitiva objetiva; la segunda, una filosofía genitiva subjetiva de la que ya hemos adelantado algunas de sus notas (si bien no las hemos identificado como tal) vinculada directamente con un elemento central del que no hemos hablado hasta ahora pero que entendemos fundamental: no podemos pensar en una filosofía de la agronomía sin una filosofía de la agricultura. Más allá de las diferencias de fondo o de forma que existen entre una y otra, la filosofía de la agronomía estaría incompleta sino incluyera dentro de su reflexión un profundo estudio y análisis, un ejercicio serio de comprensión del sentido mismo de la agricultura, del significado que tiene como actividad constitutiva civilizatoria, el que juega en cada cultura y lo que representa para quienes participan en los proyectos agronómicos. Este segundo camino significa, por consiguiente, atender a la filosofía, al significado y sentido de la agronomía y la agricultura para cada agente o comunidad que participa de su desarrollo. A modo de ejemplo, así como con el término "filosofía de la religión" podemos hacer referencia tanto a aquella rama de la filosofía que tiene por objeto de estudio el fenómeno religioso (en general o en alguna de sus manifestaciones), o hacer referencia a la filosofía de una religión determinada, en nuestro caso entendemos que la filosofía de la agronomía debería también recoger ambas dimensiones. Sólo a partir de todos éstos elementos sería posible entendemos- el análisis y la comprensión totalizadora de la cuestión agrícola.

Como se puede percibir, nuestra propuesta intenta ir más allá de la mera reproducción de los modelos clásicos de la filosofía de la ciencia bajo los cuáles la actividad filosófica debería quedar reducida al análisis puramente epistemológico, ontológico y metodológico de las distintas disciplinas y campos científicos que participan en la investigación, formación y extensión agrícola. Invita - por el contrario- a centrar el estudio en el análisis filosófico de la actividad agronómica (lo que algunos 
autores han llamado Filosofía de la ciencia real). Es decir, a entender el estudio de la actividad agrícola y agronómica como un fenómeno complejo en el que las propuestas científicas y tecnológicas se ven sometidas y condicionadas por una gran cantidad y variedad de elementos, valores, principios, bienes e intereses externos. Eso significa, en términos por ejemplo de Hugh Lacey, que a la hora de hablar de ciencias, y nosotros añadiríamos sobre los proyectos y actividades agronómico-agrícolas, debemos distinguir los valores y bienes cognoscitivos subyacentes, a la perspectiva epistemológica desde la que se piensan y son puestos en marcha, así como también de la multiplicidad de valores sociales que no sólo subyacen, sino que son claves en la planeación y desarrollo de dichos programas. En otras palabras, esta filosofía genitiva -volviendo a Bueno- debe dar cabida al desarrollo en su seno de proyectos de investigación filosófica que pongan de relieve los aspectos subjetivos que condicionan los programas agrícolas.

\section{Una filosofía de la agronomía ¿para qué? Posibles aportaciones}

Llegados a este punto es probable que aún haya quien se pregunte porqué creemos que es necesaria la constitución formal de una filosofía de la agronomía; qué aportaría ésta que no hicieran ya otras disciplinas científicas (como la historia o la sociología de la agronomía) o filosóficas (como la filosofía de la ciencia en general) o cuál es su relación con la filosofía de la biología. Vayamos por pasos.

Para empezar, entendemos que una primera razón para la constitución formal de una filosofía de la agronomía la encontramos en la misma historia, tanto de la filosofía como de la misma agronomía. La actividad agrícola, su importancia, sentido y desarrollo ha sido objeto de estudio y reflexión constante a lo largo de la historia de la filosofía desde los antiguos geopónicos.

En segundo lugar, la existencia durante el proceso constitutivo de la agronomía de problemáticas y cuestiones típicamente filosóficas (como el surgimiento mismo de la ciencia agronómica, su vinculación con problemáticas como el progreso y la existencia o no una metodología o serie de metodologías apropiadas) y la insuficientemente atención recibida por parte de los filósofos entonces y ahora parecen ameritar o reclamar atención.

En tercer lugar, hoy en día la inseguridad alimentaria vinculada con el crecimiento poblacional y los regímenes de producción y comercialización a gran escala, el control de la calidad de los alimentos (uno de los principales problemas con los que se enfrentan hoy la agricultura china), el agotamiento o reducción de recursos naturales y de la biodiversidad y la contaminación son, entre otros muchos factores y cuestiones, problemáticas que reclaman la atención de la filosofía. Pero, ¿no se está haciendo esto ya? Por supuesto, sabemos de los problemas antes descritos gracias a una extraordinaria producción de trabajos de gran calidad, entre los que podemos señalar los históricos de Joseph Cotter (1994, 2003 y 2005), los empíricos llevados a cabo desde los llamados Estudios de Ciencia y la Tecnología de Sheila Jasanoff (2011, 2004 y 2006), el análisis de las controversias políticas que rodean los actuales cambios en los sistemas de producción de alimentos y la capacidad de las comunidades y de los movimientos sociales en la toma de decisiones sobre el cambio tecnológico de Abby Kinchy (2012) o los estudios sobre los sistemas de control, estabilización e interrupción de los mercados y regulaciones transnacionales de los organismos modificados genéticamente (OGM) en la UE de Javier Lezaun (2003). Lo mismo podemos decir de las obras de Rachel Carson ([1962]2013), La primavera silenciosa y Enrique Leff (2002), Ética, vida y sustentabilidad y (2004), Racionalidad ambiental. La apropiación social de la naturaleza, o los trabajos de ecofeministas y de las diversas Propuestas del Buen Vivir. Se trata de trabajos que no sólo han llamado la atención sobre la necesidad de repensar y cambiar nuestras formas de producción agrícola, los bienes e intereses ocultos que están en juego detrás de estos modelos y muchas otras problemáticas de orden social, político y económico. Son también trabajos serios y rigurosos que han sacado estas cuestiones del terreno puramente académico y científico, haciendo que formen parte de las agendas políticas y mediáticas así como recurrentes en conversaciones comunes. Por lo tanto, ¿no es suficiente con las aportaciones hechas desde otras áreas o disciplinas? Consideramos que si bien son necesarios, son insuficientes. Por un lado, al igual que los 
estudios culturales de la ciencia no agotan en absoluto la filosofía de la física o de la biología, pensamos que ocurriría lo mismo con la filosofía de la agronomía. Sobre todo, cuando las reflexiones sobre aspectos o perspectivas clásicas de la filosofía -como los compromisos epistemológicos o implicaciones éticas subyacentes en los programas agronómicos a estudiar recordando la propuesta de Thompson (2008)- han sido poco menos que ignoradas o han quedado reducidas hasta ahora a simples apuntes marginales en estudios sociales de la ciencia. Aun reconociendo la extraordinaria importancia de ese tipo de trabajos, entendemos que no agotan o llegan a profundizar completamente en las problemáticas filosóficas. Retomando brevemente a modo de ejemplo nuestro proyecto de investigación, entendemos fundamental poner al descubierto la concepción de ciencia, de explicación científica, de experimentación y validación en torno a la cual se puso en marcha el Programa Agrícola México. Asimismo es capital qué entendían por agricultura y agronomía tanto en términos cognitivos como sociales (volviendo a retomar la distinción de Lacey) los principales agentes que participaron en el diseño y la puesta en marcha de los programas agrícolas de la Fundación Rockefeller. Para tal fin, una de nuestras primeras tareas a llevar a cabo ha sido la identificación de aquellos agentes que dan soporte e identidad al proyecto y los valores, las perspectivas y elementos más importantes (comunes) sobre los que se levantó la cosmovisión dentro del cual se pensó y puso en marcha dicho proyecto. Elementos que, como señala John Losee (2004, pp. 11-14), están basados en o condicionan el mismo desarrollo de la ciencia. Si bien, señala este autor, el análisis y clarificación de conceptos son tareas centrales de la filosofía de la ciencia, no pueden hacerse al margen del marco "ideológico", "cosmológico" y epistemológico dentro del cual se desarrolla el proyecto o grupo.

En cuarto lugar, estimamos que la filosofía de la agronomía podría ir más allá y hacer aportaciones a las mismas ciencias agronómicas tal como lo hace la filosofía de la biología, que además de aportar respuestas a cuestiones propiamente filosóficas, como las epistemológicas, desde ella es posible plantear e intentar dar respuesta a cuestiones científicas y hacer importantes contribuciones al desarrollo de teorías o paradigmas, como el evolutivo. De igual forma, la filosofía de la agronomía podría no sólo ayudar al desarrollo de la agronomía, a esclarecer la comprensión, práctica o desarrollo de las disciplinas que la constituye o de sus proyectos particulares, también podría apoyar a la filosofía de la biología. Siguiendo con el ejemplo de la teoría evolutiva debemos recordar que ésta estuvo apoyada inicialmente en las experiencias llevadas a cabo en granjas y establos y que, los primeros "soportes" teóricos, se llevaron a cabo desde "epistemologías" agrícolas. La historia y filosofía de la agronomía puede reabrir en ese sentido nuevos y ricos debates al poner de manifiesto y estudiar desde nuevas ópticas el hecho de que fuera un agrónomo estadounidense (William J. Spillman) el que "recuperara" y aplicara las leyes de Mendel antes que los europeos las redescubrieran, o que el surgimiento de la teoría de la evolución de Darwin pueda ser revisada a la luz de sus trabajos sobre la domesticación de animales y plantas, entre los que cabe destacar su obra de 1868 (Purroy 2009). Aún más, la filosofía de la agronomía pudiera incluso hacer importantes aportaciones a otras disciplinas, como es el caso de la historia agraria ¿Cómo? Si bien, somos conscientes del presentismo que inunda siempre la investigación histórica, la revisión bibliográfica realizada hasta la fecha refleja un uso excesivo de anacronismos en el uso de términos, conceptos o propuestas. Desde la definición de términos básicos como agricultura, agronomía, botánica o fitotecnia, hasta conceptos más complejos como el de progreso, deberían pasar por el análisis conceptual apuntado inicialmente. Pero más importante puede ser aún el estudio del proceso mismo de constitución de la agronomía o agricultura científica. Hay desde luego ya muchos trabajos importantes, monografías y artículos en revistas especializadas en historia de la agricultura y la agronomía, sin embargo, no encontramos reflexiones, estudios profundos y sistemáticos que aborden cuestiones tan importantes como el proceso paralelo de constitución científica de los distintos campos o disciplinas de los que se alimenta la agricultura científica, o qué debemos entender por agricultura científica, qué distingue ésta de la agricultura griega, romana, medieval e incluso renacentista e ilustrada. Se nos dirá en ese sentido, por ejemplo, que la agronomía (o nueva agricultura científica), no se basaba ya en una suerte de tratados especulativos, recolección de experiencias o conjuntos de recomendaciones como las que podemos encontrar en los trabajos de Catón el Viejo ([II a.C.] 2009), Virgilio ([29 a.C.]1963), Columela ([42 d.C.] 1879) o Gabriel Alonso de 
Herrera (([1513]1818). Sin embargo, debemos apuntar que dichos tratados y trabajos sobre agricultura que hoy diríamos "pre-científica", intentaron no sólo resumir, sistematizar o aconsejar, sino también teorizar sobre la práctica agrícola a partir de los conocimientos científicos de la época. Es decir, se intentó hacer ciencia agrícola a partir -recurriendo a Thomas Kuhn- del paradigma vigente en cada época. Incluso, encontramos otro punto común entre la literatura de la nueva agricultura científica y los tratados previos: uno de los principales objetivos en unos casos y otros era, junto a la teorización, explicación y predicción, mejorar la práctica agrícola "educando científicamente" al agricultor.

Como podemos ver, a poco que empecemos a sumergirnos, empiezan a surgir cuestiones que nos llevan desde la historia o los estudios sociales y culturales de la ciencia, al ámbito puramente filosófico, como lo es la constitución de la misma disciplina y su alcance (tanto de la agronomía en sí como de la misma filosofía de la agronomía), qué entender por ciencia, qué distingue la ciencia moderna (decimonónica, positivista) de las ciencias previas o cómo fue el proceso que llevó de unas a la otra. Sabemos que cuestiones de este tipo conducen a repetir en parte debates y reflexiones vividos en otras áreas, pero entendemos que hacerlo de manera especializada, atendiendo a la historia y naturaleza de la disciplina, puede ser importante tanto para la comprensión y desarrollo de la misma, como para la misma filosofía de la ciencia y otras disciplinas afines.

\section{Conclusiones y propuesta final}

Esperamos que estas líneas no sólo funjan como una llamada de atención sobre la necesidad de constituir una filosofía de la agronomía fuertemente vinculada con -y en parte dependiente de- la filosofía de las ciencias de la vida, sino que sirvan también para poner en marcha dicho proyecto de investigación. Un proyecto que, entendemos, debería comenzar -y este es nuestro primer y principal llamado- por la constitución de los primeros grupos, las primeras sociedades o asociaciones especializadas. Unas sociedades que pusieran en marcha los primeros encuentros y congresos específicos sobre la temática y en torno a los cuales pudieran surgir las primeras publicaciones especializadas. Con tiempo, paciencia y trabajo, nos gustaría ver cómo, fruto de estos esfuerzos, surgen cátedras y cursos profesionales de la disciplina con sus respectivos libros de texto y apoyo.

Es posible que llegados a este punto haya quien no vea aun suficientemente demostrado la pertinencia de esta propuesta, que no quede completamente demostrado qué aportaría más allá de los estudios culturales de la ciencia o que la propuesta sea incompleta o imprecisa por no apuntar, por ejemplo, hacia un marco teórico concreto. Sin embargo, consideramos -como ya apuntamos inicialmente- que no es el momento de restar, sino de sumar, no es el momento de decir si esta filosofía debería ser analítica, histórica o materialista ni de dar una estructura definitiva. Entendemos, por el contrario, que todas y cada una de las perspectivas y estudios filosóficos serían en principio necesarios y pertinentes. En términos de I. Lakatos, nos encontraríamos en un momento verificacionista, y no falsacionista, en el que los distintos trabajos particulares participarían en la constitución tanto de las heurísticas positivas como de una suerte de cinturón protector, a la par que introducirían criterios epistemológicos y recursos que la fueran robusteciendo.

Para concluir, esperamos que, más allá de la incompleta articulación de la propuesta o convencimiento generalizado sobre la necesidad y pertinencia del análisis crítico y filosófico sobre los aspectos y compromisos epistemológicos, ontológicos, metodológicos u éticos subyacentes en la actividad agronómica, estas líneas contribuyan en la generación de un espacio para el debate y al diálogo que es a lo que la propuesta de este número especial de la revista Metatheoria -entendemosinvita.

Bibliografía

Alexander, A. (2008), "Different Paths, same Mountain: Daoism, Ecology and the New Paradigm of Science”, 
International Journal of Green Economics 2(2): 153-175.

Alonso de Herrera, G. ([1513]1818), Agricultura General, Vol. I, Madrid: Imprenta Real.

Berger, A. (1995), "Is Agricultural Research in Africa Worthwhile Today?", en Berger, A. (ed.), Twice HumanityImplications for Local and Global Resource Use, Stockholm: The Nordic African Institute and Forum for Development Studies Uppsala, pp. 65-75.

Brown, L.R. (1981), "World Food Resources and Population: The Narrowing Margin”, Population Bulletin 36(3): 1-44.

Binkley, D. y R.F. Fisher (2013), Ecology and Management of Forest Soils, $4^{\mathrm{a}}$ ed., Chichester: Wiley-Blackwell.

Bueno, G. (2004), La vuelta a la caverna. Terrorismo, guerra y globalización, Madrid: Ediciones B.

Bunge, M. (2004), “QQué es y para qué sirve la epistemología?”, en Bunge, M., Epistemología, México: Siglo XXI, pp. 21. 33.

Carson, R. ([1962]2013), Primavera sileciosa, Barcelona: Booket.

Carter, L. (2007), "A Case for a Duty to Feed the Hungry: GM Plants and the Third World", Science and Engineering Ethics 13(1): 69-82.

Cassini, A. (2013), "Sobre la historia de la filosofía de la ciencia. A propósito de un libro de C. Ulises Moulines", Crítica 45(134): 69-97.

Catón, M.P. ([II a.C.] 2009), De Agri Cultura, Madrid: Tecnos.

Clarke, J. (2010), The Ordinary People of Essex: Environment, Culture, and Economy on the Frontier of Upper Canada, Montreal: McGill-Queen's University Press.

Cohen, B. (2009), Notes from the Ground: Science, Soil, and Society in the American Countryside, New Haven: Yale University Press.

Columela, L.J.M. ([42 d.C.] 1879), Los doce libros de agricultura, Madrid: Imprenta de Miguel Ginesta. Versión digital: http://bdh-rd.bne.es/viewer.vm?id=0000011708\& page=1.

Cotter, J. (1994), "The Origins of the Green Revolution in Mexico. Continuity or Change?" en Rock, D. (ed.), Latin America in the 1940s. War and Postwar Transitions, Berkeley: University of California Press, pp. 224-247.

Cotter, J. (2003), Troubled Harvest: Agronomy and Revolution in Mexico, 1880-2002, Westportt: Praeger.

Cotter, J. (2005), "Cultural Wars and New Technologies: The Discourse of Plant Breeding and the Professionalisation of Mexican Agronomy, 1880-1994”, Science, Technology \& Society: 141-168.

DeWalt, B.R. (1978), "Appropriate Technology in Rural Mexico: Antecedents and Consequences of an Indigenous Peasant Innovation", Technology and Culture 19: 32-52.

Egerton, F.N. (2012), Roots of Ecology. Antiquity to Haeckel, Berkeley: University of California Press.

Elena, A. y J. Ordóñez (1996), "De la revolución científica a la revolución industrial. La dimensión tecnológica del newtonianismo", Hispania: Revista Española de Historia 56(193): 541-564.

Jasanoff , S. (2011), "Constitutional Moments in Governing Science and Technology", Science and Engineering Ethics 17(4): 621-638.

Jasanoff, S. (2004), States of Knowledge: The Co-Production of Science and Social Order, London-New York: Routledge.

Jasanoff, S. (2006), "Biotechnology and Empire: The Global Power of Seeds and Science", Osiris 21(1): 273-292.

Kinchy, A. (2012), Seeds, Science, and Struggle: The Global Politics of Transgenic Crops, Cambridge, MA: The MIT Press.

Klaassen, J.A. (2007), “Contemporary Biotechnology and the new 'Green Revolution': Feeding the World with 'Frankenfoods?', Social Philosophy Today: Science, Technology, and Social Justice 22: 103-113.

Lacey, H. (1998), Valores e atividade cientifica, São Paulo: Editora Discurso.

Lazo-Flores, T. (2012), "Grading Green: Strands of Sustainability Theory and Practice in Interior Design Courses", Sustainability 5(5): 323-330.

Leff, E. (2002), Ética, vida y sustentabilidad, Serie Pensamiento Ambiental Latinoamericano, México: PNUMA.

Leff, E. (2004), Racionalidad ambiental. La apropiación social de la naturaleza, México: Siglo XXI. 
186 | Francisco Javier Serrano-Bosquet y Eva Luisa Rivas Sada

Lezaun, J. (2003), Policing purity: Testing, Traceability, and the Governance of Genetically Modified Organisms, Tesis doctoral, Ithaca: Cornell University.

Light, A. y A. Wallace (2005), "Not out of the Woods: Preserving the Human in Environmental Architecture", Environmental Values 14(1): 3-20.

Loftin, R. (1993), "The Violence of the Green-Revolution - Third World Agriculture, Ecology, and Politics Shiva,V", Journal of Value Inquiry 27(3-4): 559-526.

Losee, J. (2004), Introducción histórica a la filosofía de la ciencia, Madrid: Alianza.

Noll, S. (2013), "Broiler Chickens and a Critique of the Epistemic Foundations of Animal Modification", Journal of Agricultural and Environmental Ethics 26(1): 273-280.

Purroy, J. (2009), "Variaciones del Darwin doméstico. Reflexiones en torno a 'La variación en animales y plantas domésticos”, Métode 60: 195-199.

Reichenbach, H. (1938), Experience and Prediction: An Analysis of the Foundations and the Structure of Knowledge, Chicago: The University of Chicago Press.

Scott, D. (2011), "The Technological Fix Criticisms and the Agricultural Biotechnology Debate”, Journal of Agricultural and Environmental Ethics 24(3): 207-226.

Serrano-Bosquet, F. y G. Caponi (2014), "Warren Weaver y el Programa de Biología Experimental de la Fundación Rockefeller”, Scientiæ Studia 12(1): 137-167.

Shrader-Frechette, K. (2005), "Property Rights and Genetic Engineering: Developing Nations at Risk", Science and Engieneering Ethics 11(1): 137-149.

Shurtz, N. (2012), "Eco-Friendly Building from the Ground up: Environmental Initiatives and the Case of Portland, Oregon", Journal of Environmental Law and Litigation 27(1): 237-362.

Thompson, P. (2008), "The Agricultural Ethics of Biofuels: A first look”, Journal of Agricultural and Environmental Ethics 21(2): 183-198.

Thompson, R.P. (2011), Agro-Technology: A Philosophical Introduction, Cambridge: Cambridge University Press.

Trias, M. (1987), "Population and Development”, Boletin Asociación Chilena de Protección de la Familia 23(7-12): 3-7.

Virgilio, P. ([29 a.C.]1963), Geórgicas, México: Universidad Nacional Autónoma de México. 